\title{
RNA is for activation
}

\section{By Amy Donner, Senior Editor}

Although siRNA-based therapeutics have recently been clinically validated as a strategy to knock down gene expression, a lesser-known function for short double-stranded RNAs is their ability to turn on the expression of target genes. Now, an academic team sponsored by MiNA Therapeutics Ltd. has found a way to attack liver cancer in rats by using a short activating RNA to upregulate expression of a tumor suppressor, ${ }^{1}$ and the company plans to advance the program to the clinic this year.

siRNAs target mRNA coding regions and are used to knock down gene expression via targeted cleavage by an RNA-induced silencing complex (RISC). The clinical development of this strategy was pioneered by Alnylam Pharmaceuticals Inc., which last month entered into a major collaboration to develop products with the Genzyme Corp. unit of Sanofi. ${ }^{2}$

At least 12 additional companies are pursuing the development of RNAi-based therapeutics, one of which, Dicerna Pharmaceuticals Inc., went public last week and saw its stock more than triple in its first day of trading. The preclinical-stage company ended the week with a market cap of $\$ 683.7$ million.

Small activating RNAs (saRNAs) are structurally related to siRNAs, but instead of targeting mRNA, they target regulatory regions of DNA upstream of genes of interest. This can lead to increased gene expression, though the precise mechanisms of action for saRNAs are poorly understood.

MiNA CEO Robert Habib told SciBX, "The beauty of saRNA is that well-established oligonucleotide designs can be used as triggers for gain of function. That allows MiNA to leverage years of pharmaceutical development, from assays to delivery, which have enabled a robust pipeline of 'loss-of-function therapeutics"'

These advances include the development of lipid nanoparticle formulations that enable efficient short double-stranded RNA delivery to the liver in humans.

Thus, MiNA set out to design a liver-targeted saRNA to treat hepatocellular carcinoma (HCC).

Prognosis for patients with HCC is poor because a majority have advanced disease at the time of diagnosis, which impairs liver function and prevents surgery. Thus, therapeutic strategies that antagonize tumor formation and improve or preserve liver function are needed.

The company homed in on CCAAT enhancer binding protein- $\alpha$
(CEBPA), a tumor suppressor that is also known to upregulate genes involved in hepatocyte function, such as albumin. CEBPA is expressed in functional hepatocytes in the liver but is downregulated in hepatoma cells.

"CEBPA is a transcription factor with a number of regulatory functions including controlling hepatocyte response to injury," said Habib. "Evidence suggested that these features could be translated into a promising therapeutic candidate."

John Rossi, cofounder of both Dicerna and MiNA, said that the target was attractive because it is a master regulator of gene expression for a large group of genes that alter cell proliferation.

Rossi is chair and professor of molecular and cellular biology and dean at City of Hope and is on the scientific advisory board for several companies developing RNA therapeutics including Dicerna, Arrowhead Research Corp. and Benitec Biopharma Ltd.

In a study led by Rossi and Nagy Habib-Robert Habib's fatherscientists tested whether an saRNA that upregulated CEBPA expression could be used to improve cirrhotic liver function, treat HCC or both. ${ }^{1}$ Nagy Habib is lead clinician and head of the Department of Hepatobiliary Surgery at Imperial College London.

To develop an saRNA candidate, the team used bioinformatics approaches to search databases for antisense transcripts that overlapped with CEBPA and then used a design algorithm to predict short doublestranded RNAs that might act as saRNAs by blocking the antisense transcript.

In a cultured human liver carcinoma cell line, an saRNA targeting the CEBPA promoter region doubled $C E B P A$ transcript levels, increased albumin secretion and decreased proliferation compared with scrambled saRNA. The CEBPA-saRNA also reduced DNA methylation near the CEBPA promoter, hinting at a possible molecular explanation for its activity.

To test the saRNA in vivo, the scientists conjugated it to a triethanolamine core poly(amidoamine) dendrimer that has previously been used to deliver siRNA to the liver in vivo. ${ }^{3}$

In a rat model of liver cirrhosis with HCC, the CEBPA-saRNA dendrimer increased expression of Cebpa and levels of circulating albumin and decreased activity of the liver enzymes aspartate aminotransferase (AST) and alanine aminotransferase (ALT) compared with scrambled saRNA dendrimer. Gene expression data were also consistent with improved liver function in CEBPA-saRNA dendrimertreated rats.

The CEBPA-saRNA dendrimer also decreased tumor formation and led to a $10 \%$ decrease in the expression of a preneoplastic marker.

The study was published in Hepatology and included scientists from the Norwegian University of Science and Technology, National Taiwan University, Marseille Interdisciplinary Center for Nanoscience, General University Hospital of Larissa, Biomedical Research Foundation of the Academy of Athens, University of California, Los Angeles, High Technology Medical Center, Tbilisi 
State Medical University, Qatar Biomedical Research Institute and University of Pennsylvania. It was funded by MiNA.

The devil is in the details

Although researchers contacted by $S c i B X$ agreed that upregulating CEBPA holds promise in HCC, they were concerned by the lack of a clear molecular explanation for saRNA action.

Jim Barsoum, CSO of RaNA Therapeutics Inc., said that there were many possible ways the saRNA could be acting. "These saRNAs could be blocking the activity or inducing the degradation of antisense transcripts that repress transcription. The saRNA may be modulating the recruitment of histone methyltransferases or demethylases that naturally lead to the repression of transcription. Some data suggest that an RNAi-mediated mechanism may be responsible," he said.

RaNA is developing short single-stranded oligonucleotides to selectively activate target gene expression. One platform is based upon the ability of these oligonucleotides to sterically block the interactions between RNA and polycomb repressive complex 2 (PRC2).

"Current drug development strategies place great value on having a thorough mechanistic understanding," added Barsoum.

Claes Wahlestedt agreed. "saRNAs are arguably controversial, and the underlying mechanism of CEBPA-saRNA is almost entirely unclear," he said. He added that he would be more confident about the therapeutic strategy if the mechanism was better understood.

Wahlestedt is associate dean for therapeutic innovation, director of the Center for Therapeutic Innovation and a professor of psychiatry and behavioral sciences at the University of Miami Miller School of Medicine. He also cofounded Curna Inc. to commercialize a distinct single-stranded noncoding RNA-based strategy for upregulating gene expression. Opko Health Inc. acquired Curna in 2011.

MiNA acknowledges the need to clarify the mechanism. "The exact mechanism of transcriptional gene activation is still being defined," said Robert Habib. He indicated that some general features about saRNA action have been established, and MiNA has evidence from chromatin immunoprecipitation assays that CEBPA-saRNA interacts specifically with the transcription start site of the CEBPA promoter.

Rossi added that a noncoding RNA is likely involved. "We believe that an saRNA-long noncoding RNA complex recruits chromatin-remodeling enzymes, resulting in positive histone marks and also recruiting RNA polymerase to the targeted promoter."

A noncoding RNA-mediated mechanism for regulating CEBPA expression was published last year in Nature by an independent group from Harvard Medical School. ${ }^{4}$ The scientists reported a noncoding RNA — called ecCEBPA — derived from CEBPA that binds DNA (cytosine5-)-methyltransferase 1 (DNMT1), preventing methylation of the gene and increasing expression of CEBPA. MiNA is investigating the relationship between ecCEBPA and their CEBPA-saRNA.

\section{A clinical formulation}

MiNA has now begun clinical testing of a nanoparticle-based formulation of a CEBPA-upregulating saRNA, MTL-501. "MTL-501 is in a first-in-human dose-escalation study," said Robert Habib. MiNA anticipates initiating a Phase I study in the U.K. in under a year.

MTL-501 is an optimized version of the published CEBPA-saRNA dendrimer.

Barsoum and Wahlestedt both noted that formulating and manufacturing CEBPA-saRNA is not trivial because double-stranded RNA is more difficult to deliver than single-stranded RNA.

"Although other published studies have shown this type of nanoparticle delivery vehicle to be safe, the complex creates manufacturing challenges and could lead to its own set of toxicities," said Barsoum.

"Using dendrimers for delivery is not uncomplicated," added Wahlestedt.

However, Rossi told SciBX that MiNA is moving forward to ramp up production. "We know from our preclinical animal work that this is a safe and efficacious approach for treating liver cancer and fatty liver disease, so we would like to scale up manufacturing of the components for a Phase I human trial," he said.

Robert Habib said that the CEBPA-saRNA has been tested in a range of subcutaneous tumor xenograft and fatty liver disease models in addition to the published work in the rat model of cirrhosis with HCC.

The product and its method of use are covered by MiNA's patent portfolio, and MTL-501 is available for partnering. MiNA is also developing additional saRNA therapeutic candidates. "Now that loss-of-function oligonucleotide-based strategies have been substantially derisked, gain of function is a strikingly obvious next step," Robert Habib said.

Barsoum agreed. "For many years, oligonucleotides have been viewed as a means to decrease gene expression. There are now multiple examples of oligonucleotides being used to increase gene expression," he said. "We now are realizing that oligonucleotides are a rich and diverse therapeutic modality that can possess a wide variety of different activities."

Donner, A. SciBX 7(5); doi:10.1038/scibx.2014.136 Published online Feb. 6, 2014

\section{REFERENCES}

1. Reebye, V. et al. Hepatology; published online Dec. 9, 2013; doi:10.1002/hep.26669

Contact: Nagy A. Habib, Imperial College London, London, U.K. e-mail: nagy.habib@imperial.ac.uk

Contact: Vikash Reebye, same affiliation as above e-mail: v.reebye@imperial.ac.uk

2. McCallister, E. BioCentury 22(3), A1-A5; Jan. 20, 2014

3. Zhou, J. et al. Mol. Ther. 19, 2228-2238 (2011)

4. Di Ruscio, A. et al. Nature 503, 371-376 (2013)

\section{COMPANIES AND INSTITUTIONS MENTIONED}

Alnylam Pharmaceuticals Inc. (NASDAQ:ALNY), Cambridge, Mass. Arrowhead Research Corp. (NASDAQ:ARWR), Pasadena, Calif. Benitec Biopharma Ltd. (ASX:BLT), Balmain, New South Wales, Australia

Biomedical Research Foundation of the Academy of Athens, Athens, Greece

City of Hope, Duarte, Calif.

Dicerna Pharmaceuticals Inc. (NASDAQ:DRNA), Watertown, Mass.

General University Hospital of Larissa, Larissa, Greece

Genzyme Corp., Cambridge, Mass.

Harvard Medical School, Boston, Mass. 


\section{ANALYSIS}

High Technology Medical Center, Tbilisi, Georgia Imperial College London, London, U.K.

Marseille Interdisciplinary Center for Nanoscience, Marseille, France

MiNA Therapeutics Ltd., London, U.K.

National Taiwan University, Taipei City, Taiwan

Norwegian University of Science and Technology, Trondheim, Norway
Opko Health Inc. (NYSE:OPK; Tel Aviv:OPK), Miami, Fla. Qatar Biomedical Research Institute, Doha, Qatar RaNA Therapeutics Inc., Cambridge, Mass. Sanofi (Euronext:SAN; NYSE:SNY), Paris, France Tbilisi State Medical University, Tbilisi, Georgia University of California, Los Angeles, Calif. University of Miami Miller School of Medicine, Miami, Fla. University of Pennsylvania, Philadelphia, $\mathrm{Pa}$. 